\title{
Generation of Ultrasound Based on the Frequency Response Characteristics of the "Koss Pro Headphone" with R. David Case Sound Wave Files-A Case Study
}

\author{
R. David Case1, D. Chuckravanen² \\ ${ }^{1}$ The R. David Case Sound Device and Research Collaborator, Van Buren, Missouri, USA \\ ${ }^{2}$ British Association of Cognitive Neuroscience, Department of Psychology, University of Essex, Colchester, \\ United Kingdom \\ Email: dineshen2013@gmail.com
}

How to cite this paper: Case, R.D. and Chuckravanen, D. (2021) Generation of Ultrasound Based on the Frequency Response Characteristics of the "Koss Pro Headphone" with R. David Case Sound Wave Files'-A Case Study. World Journal of Engineering and Technology, 9, 374-383.

https://doi.org/10.4236/wjet.2021.92026

Received: February 15, 2021

Accepted: May 24, 2021

Published: May 27, 2021

Copyright ( 2021 by author(s) and Scientific Research Publishing Inc. This work is licensed under the Creative Commons Attribution International License (CC BY 4.0).

http://creativecommons.org/licenses/by/4.0/

\begin{abstract}
The research aim of this work is to analyse the characteristics of R. David Case Sound waves that seemed to have positive effects on people suffering from tinnitus. Moreover, the participants who listened to these sound wave files using specific headsets or headphones of the Koss models (ktx Pro, ksc-75) showed improved health condition such as alleviating tinnitus. Therefore, these discoveries have encouraged R. David Case to pursue positively his journey into finding out what is special about those sounds when being listened specifically with Koss models. In this research, we focus mainly on the technical aspects of R. David Case sound signals which he has recorded. These specific sound waves were analysed using time domain, frequency domain as well as the effect of using the Koss Pro headphone frequency response characteristics applied to the sound. Results obtained from the analysis demonstrated that the generation of ultrasound can be the underlying reason for the treatment of the tinnitus.
\end{abstract}

\section{Keywords}

Ultrasound, Tinnitus, David Case Sound Wave File, Frequency Analysis, Healing

\section{Introduction}

In General, hearing depends on a sequence of complicated stages that change sound waves in the air into electrical signals. Then, the auditory nerve transports 
these signals to our brain. The sound wave enters the outer ear and then it travels through the ear canal leading to the eardrum. Subsequently, the eardrum vibrates owing to the incoming sound waves and then sends these vibrations to tiny bones that are found in the middle ear. These bones amplify the sound vibrations which are then sent to the cochlea (snail-shaped structure) filled with fluid in the inner ear. An elastic partition runs from the beginning to the end of the cochlea, splitting it into an upper and lower part. This partition is called the basilar membrane because it serves as the base, or ground floor, on which key hearing structures sit. Once the vibrations cause the fluid inside the cochlea to ripple, a traveling wave forms along the basilar membrane, hair cells-sensory cells sitting on top of the basilar membrane-ride the wave. Hair cells near the wide end of the snail-shaped cochlea detect higher-pitched sounds, such as an infant crying. Those closer to the center detect lower-pitched sounds, such as a large dog barking. As the hair cells move up and down, microscopic hair-like projections (known as stereocilia) that perch on top of the hair cells bump against an overlying structure and bend. Bending causes pore-like channels, which are at the tips of the stereocilia, to open up. When that happens, chemicals rush into the cells, creating an electrical signal. The auditory nerve carries this electrical signal to the brain, which turns it into a sound that we recognize and understand.

The normal range of human hearing is $20 \mathrm{~Hz}$ to $20,000 \mathrm{~Hz}$. Under ideal lab conditions, human can hear sound as low as $12 \mathrm{~Hz}$ and as high as $28 \mathrm{kHz}$, though the threshold increases sharply at $15 \mathrm{kHz}$ in adults, corresponding to the last auditory channel of the cochlea. The next sub-section explains what is ultrasound and why it is important for this research work.

\subsection{What is Ultrasound?}

Ultrasound is acoustic (sound) energy in the form of waves having a frequency above the human hearing range. The highest frequency that the human ear can detect is approximately 20 thousand cycles per second $(20,000 \mathrm{~Hz})$. This is where the sonic range ends, and where the ultrasonic range begins. In physics the term "ultrasound" applies to all acoustic energy with a frequency above human hearing (20,000 hertz or 20 kilohertz). Typical diagnostic sonographic scanners operate in the frequency range of 2 to 18 megahertz, hundreds of times greater than the limit of human hearing. Ultrasound is used in electronic, navigational, industrial, and security applications. It is also used in medicine to view internal organs of the body.

Since the inception of ultrasound as a therapeutic tool, its potential to treat disorders throughout the brain has been explored. The objective has been to utilize ultrasound's focusing ability to target precisely within deep tissues, affecting only the interested volume while leaving all other structures unaltered [1]. Varying the ultrasound parameters not only allows ablation of pathological tissue, such as brain tumours and metastases, or silencing of dysfunctional neuronal circuits, but also opens up the blood-brain barrier for targeted drug deli- 
very and modulation of neural function [2]. Before we go directly into analysing those unique sound waves generated by a "device" built by R. David Case, it is important also to define what is tinnitus and how it affects a lot of people worldwide in the next sub-section.

\subsection{Tinnitus}

Tinnitus usually occurs when one experiences ringing or other noises in one or both ears (See Figure 1). The noise you hear when you have tinnitus is not caused by an external sound, and other people usually cannot hear it. In fact, tinnitus is a common problem. It affects about $15 \%$ to $20 \%$ of people. Tinnitus is usually caused by an underlying condition, such as age-related hearing loss, an ear injury or a problem with the circulatory system. For many people, tinnitus improves with treatment of the underlying cause or with other treatments that reduce or mask the noise, making tinnitus less noticeable. In this research, we have investigated how the sound files when listening from Koss Pro headphones can really pose as Anti-tinnitus. Next section describes the methodology employed to analyse the sound wave signals as well as the effect of the frequency response characteristics of the Koss Pro headphones on R. David Case sounds.

\section{Research Methodology}

All analyses are conducted on Scientific Matlab R2009a Platform running on a 64-bit computer system architecture with Intel Core I7 and processing speed is $2.90 \mathrm{GHz}$. Sound files were recorded by Mr. R. David Case and they are in *.wav format and the digital size of the sound file is about $800 \mathrm{MB}$. All sound files (stereo sounds) were imported to the Matlab environment for analyses purposes. Spectral analyses are conducted on those sound waves, and also the effect of the frequency responses of the specific Koss pro headphone on those sound files are investigated too. Experiment is being conducted on Koss Pro headphone device with the following specifications: $15 \mathrm{~Hz}$ to $25,000 \mathrm{~Hz}, 60 \mathrm{Ohms}, 101 \mathrm{~dB}$ SPL, 4 $\mathrm{ft} / 1.2 \mathrm{~m} .3 .5 \mathrm{~mm}$ (See Figure 2). In addition, follow-ups were conducted on all the people who utilised such sounds to improve their health conditions such as tinnitus, or vibrations in certain parts of the body or to reduce "voices" that only them can hear.

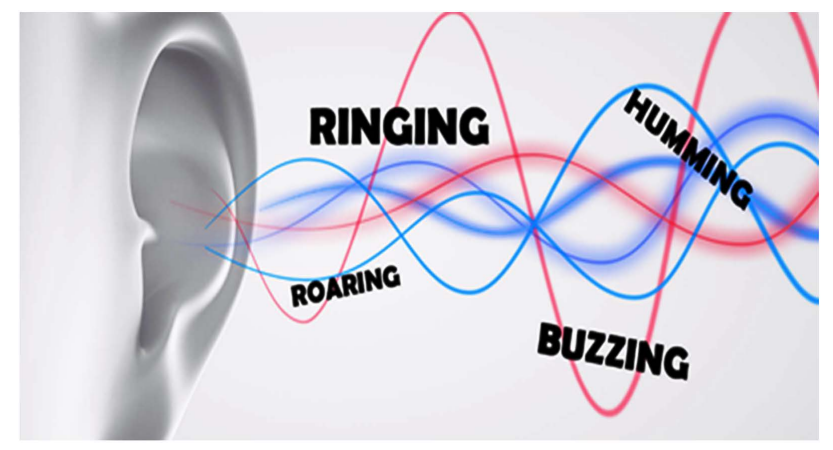

Figure 1. Tinnitus and the types of ringing. 


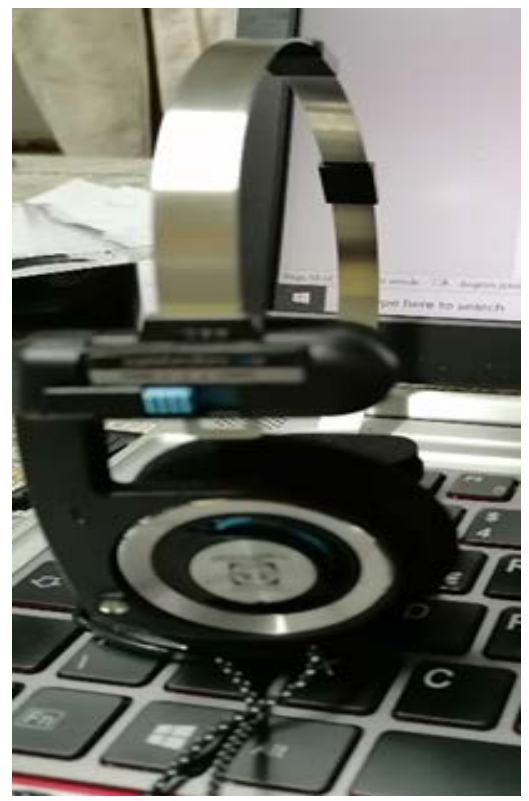

Figure 2. Koss Pro Headphone under analysis for frequency response using Matlab Software R2009a.

\section{Results}

\subsection{Time-Domain Presentation of the R. David Case Sound File}

The sound wav file was imported to the Matlab environment so as the sound wav data can be used for further analyses as the sound data is in a matrix format. Once the sound wav was in the Matlab workspace, the following Figure 3 was generated in order to visualise in the time domain the sound wav. The sampling frequency of the sound wave is $44.1 \mathrm{kHz}$.

In contrast to Figure 3 which shows that the sound is varying randomly (where we need to see what is happening in frequency domain), in Figure 4, the amplitude of the sound changes abruptly at approximately half of the time period.

\subsection{Frequency Domain Presentation of R. David Case Sound File}

As shown in Figure 4, when the spectral analysis is applied to the component 1 of the Sound wav, there were frequency peaks at around $0,0.4$, and $0.8 \times 10^{4} \mathrm{~Hz}$. On the other hand, when the spectral analysis is applied to the component 2 of the sound wav, there were frequency peaks at around $0 \mathrm{~Hz}, 0.20,0.25,0.4$ and $0.9 \times 10^{4} \mathrm{~Hz}$ (Figure 5 and Figure 6).

\subsection{Characteristic Frequency Response of Koss Pro Headphone}

From Figure 7, it is clearly observed that the power-frequency spectrum is not constant and varies throughout the normal hearing frequency spectrum. It decreases exponentially from $0 \mathrm{~Hz}$ to $4000 \mathrm{~Hz}$ and then the power increases at around 5000 $\mathrm{Hz}$ and again the power decreases to a minimum at about $7000 \mathrm{~Hz}$ and then increases to a plateau effect at around $10,000 \mathrm{~Hz}$ and decreases till to $15,000 \mathrm{~Hz}$. 


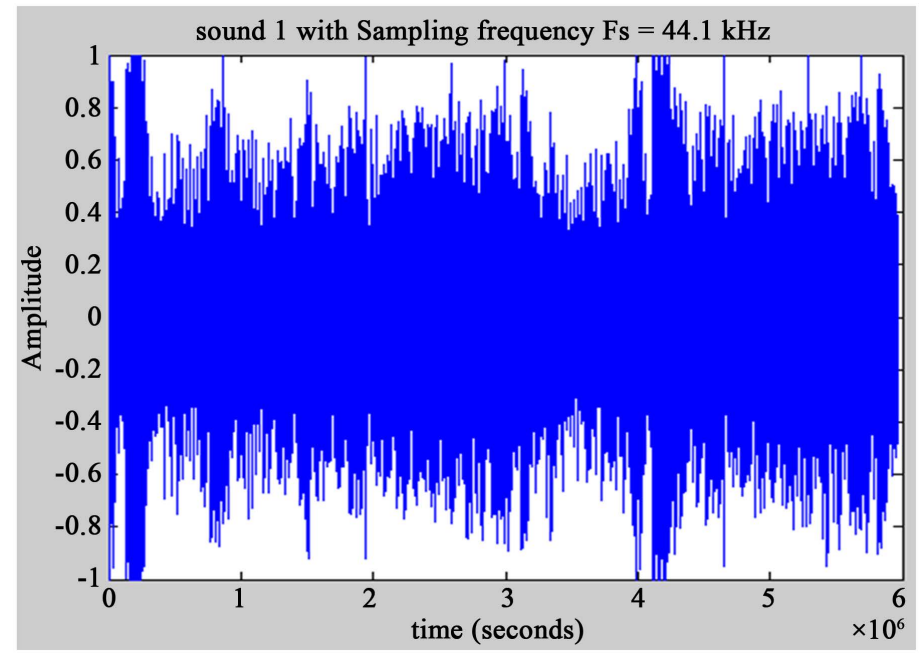

Figure 3. Sound 1 component of the device stereo sound wav (Amplitude vs. Time).

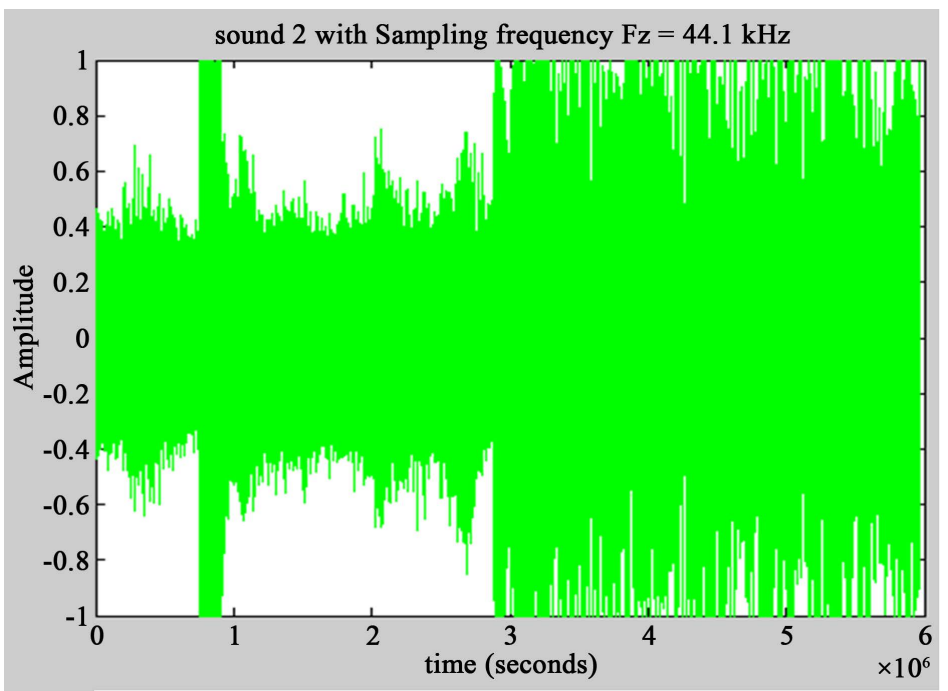

Figure 4. 2nd sound component in the stereo sound wav data (Amplitude vs. Time).

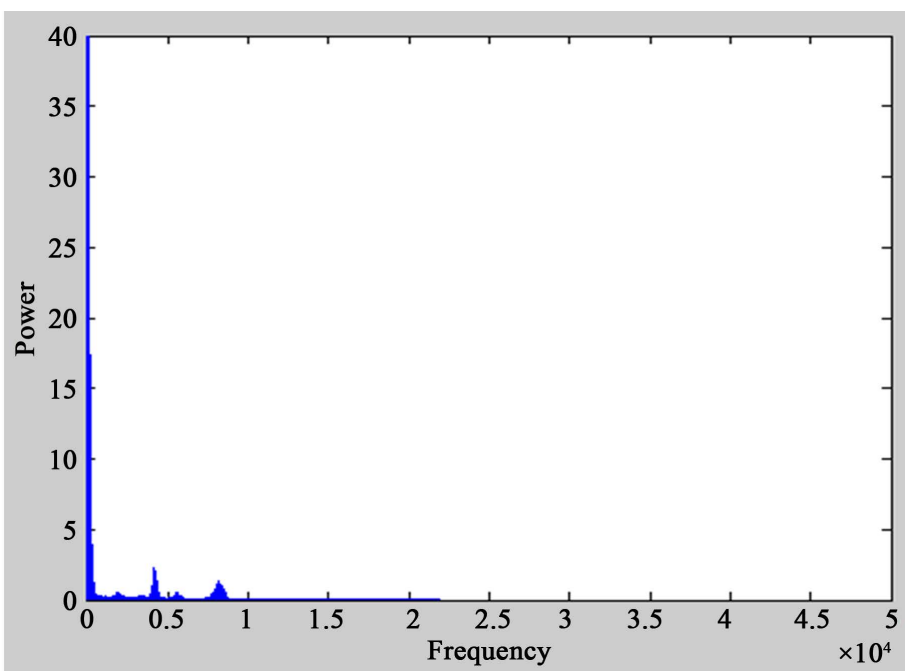

Figure 5. Spectral analysis with Frequency centred at $\mathrm{f}=0 \mathrm{~Hz}$ for sound component 1 . 


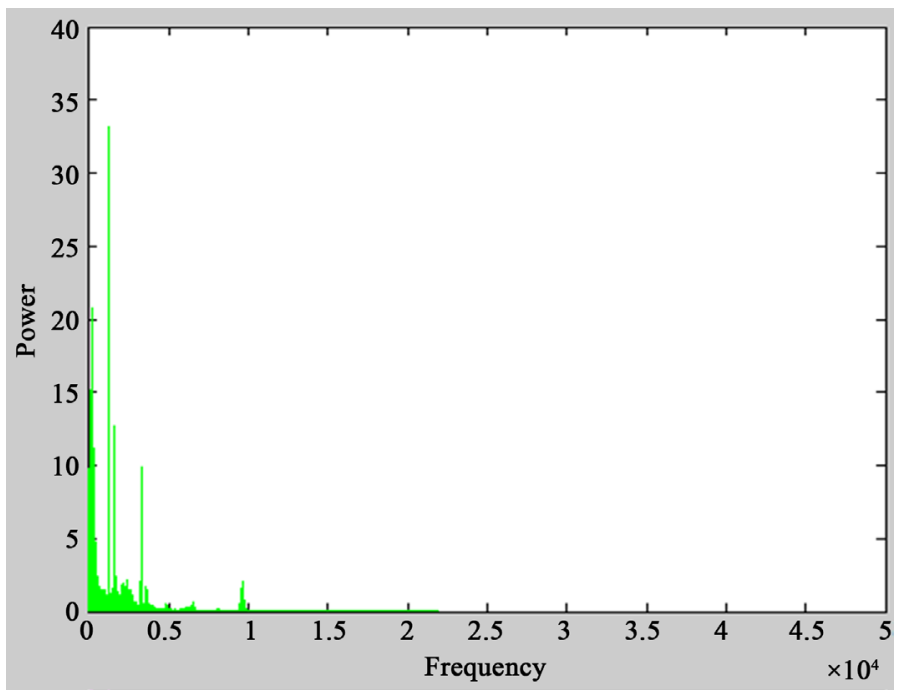

Figure 6. Power vs. Frequency spectrum (for sound component 2 of the recorded R. David Case sound wave. Frequency centred at $\mathrm{f}=0 \mathrm{~Hz}$ for Sound component 2.

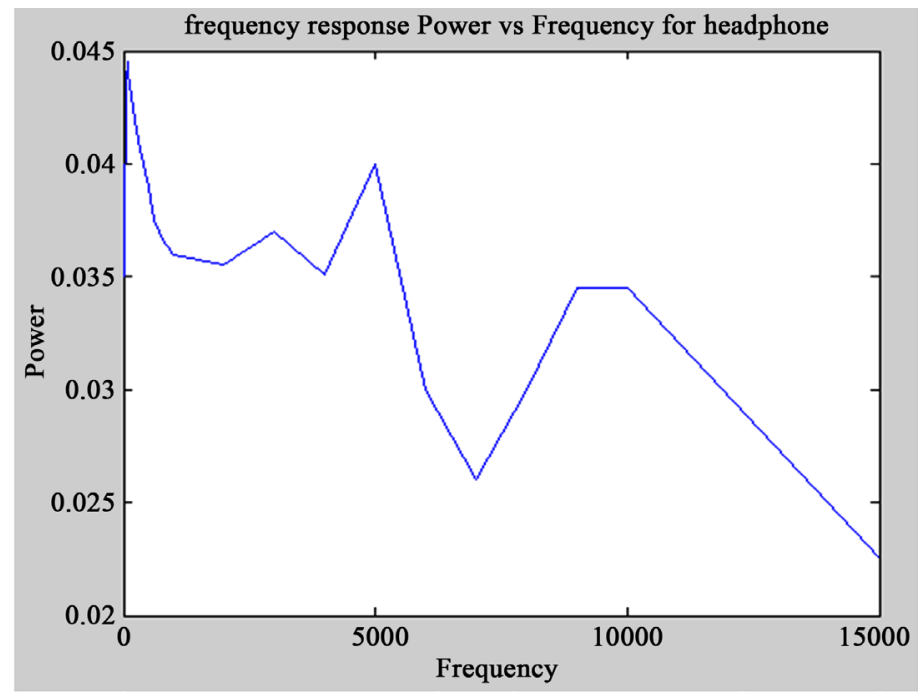

Figure 7. The power (W)-frequency $(\mathrm{Hz})$ response of the Koss Pro headphone.

\subsection{Convolution of the Sound Wave Signals with the Frequency Characteristics of the Koss Pro Headphone}

\subsubsection{Application of Convolution}

One of the most important concepts in Fourier theory is that of a convolution. Mathematically, a convolution is defined as the integral over all space of one function at $x$ times another function at $u-x$. The integration is taken over the variable $x$ (which may be a $1 \mathrm{D}$ variable), typically from minus infinity to infinity over all the dimensions. So the convolution is a function of a new variable $u$, as shown in the following equations.

$$
C(\boldsymbol{u})=f(\boldsymbol{x}) \otimes g(\boldsymbol{x})=\int_{\text {space }} f(\boldsymbol{x}) g(\boldsymbol{u}-\boldsymbol{x}) \mathrm{d} \boldsymbol{x}
$$

$$
=g(\boldsymbol{x}) \otimes f(\boldsymbol{x})=\int_{\text {space }} g(\boldsymbol{x}) f(\boldsymbol{u}-\boldsymbol{x}) \mathrm{d} \boldsymbol{x}
$$

The cross in a circle is used to indicate the convolution operation [3] [4] [5] [6]. 
Note that it does not matter which function to take first, i.e. the convolution operation is commutative. The function $f(x)$ represents the function of the sound wave and the function $g(x)$ represents the Headphone frequency response characteristics.

The positive working of the sound wave coupled with the Koss headphones produce a frequency which is healing the inner ear organ of the human body and act as an anti-tinnitus, and therefore convolution algorithm is applied to the sound wav and also the frequency response characteristics of the Koss headphone to produce a new convolution spectrum elaborated in the next sub-section.

\subsubsection{Analysed Work}

It was interesting to find that the convolved signal of the frequency response of the Koss headphone and the Sound component 1 did not show anything visually significant. However, when the convolution method was applied to the second sound wave component, the following spectrum Figure 8 is obtained.

It is clearly observed that there are maximum peaks at the frequency $2.822 \times$ $10^{6} \mathrm{~Hz}$ which is created from the machinery of the sound device and the headphone and this ultrasonic wave frequency needs to be further investigated.

\subsection{Experiment Repeated with a 80 Minute Sound Wave File}

The sound wave signals were brought to Matlab environment and as it was large size (memory), my program loops through every 2 minutes of the sounds in order to depict the various frequency components (frequency spectrum), the maximum frequency component also as well as the sounds through time. There was decrease and increase in the signal throughout the duration of the sound recordings. We have found the following peaked frequencies for each sound or rather ultra-sound component (Figure 9 and Figure 10).

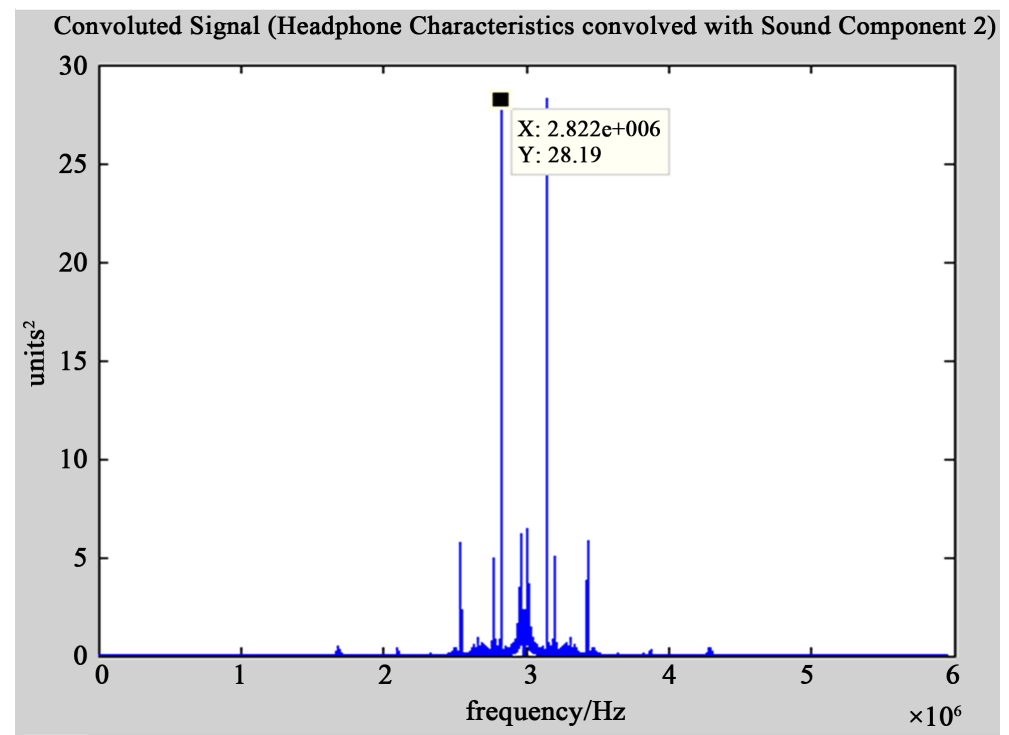

Figure 8. Spectrum of the sound wave signals when they were convoluted with the frequency response from the headphone Koss. 


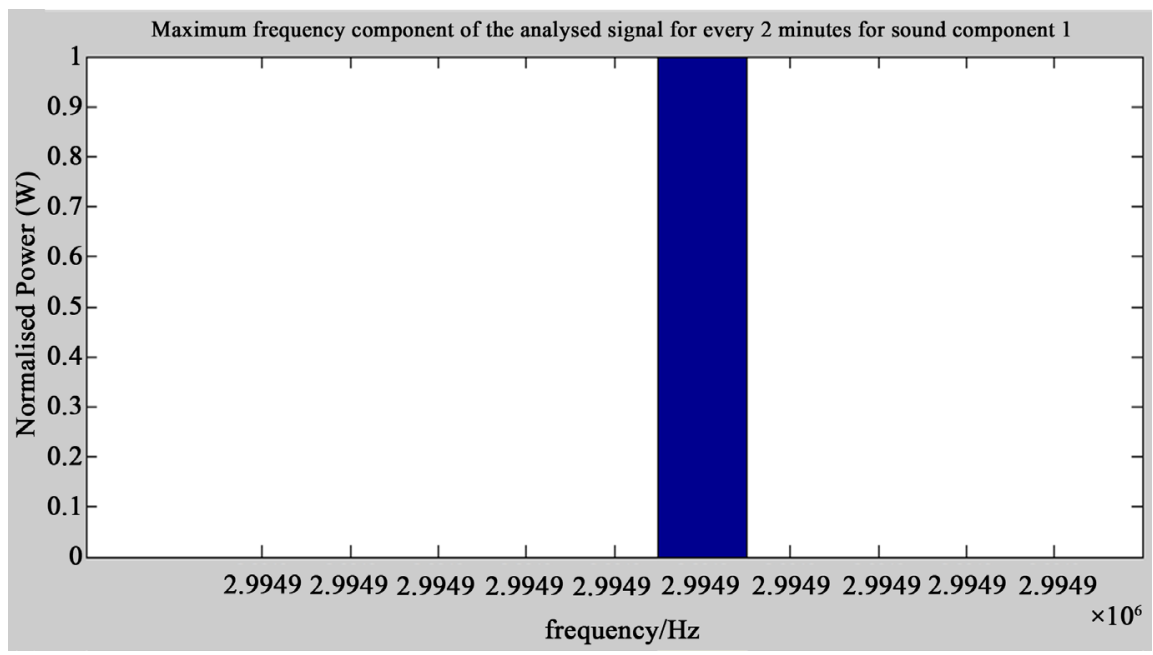

Figure 9. The frequency component after the application of Fast Fourier transform (FFT) on the signal component 1 demonstrated a maximum peak at $2.9949 \mathrm{MHz}$ for the sound component 1 and this is the average for every 2 minutes for the sound wav file.

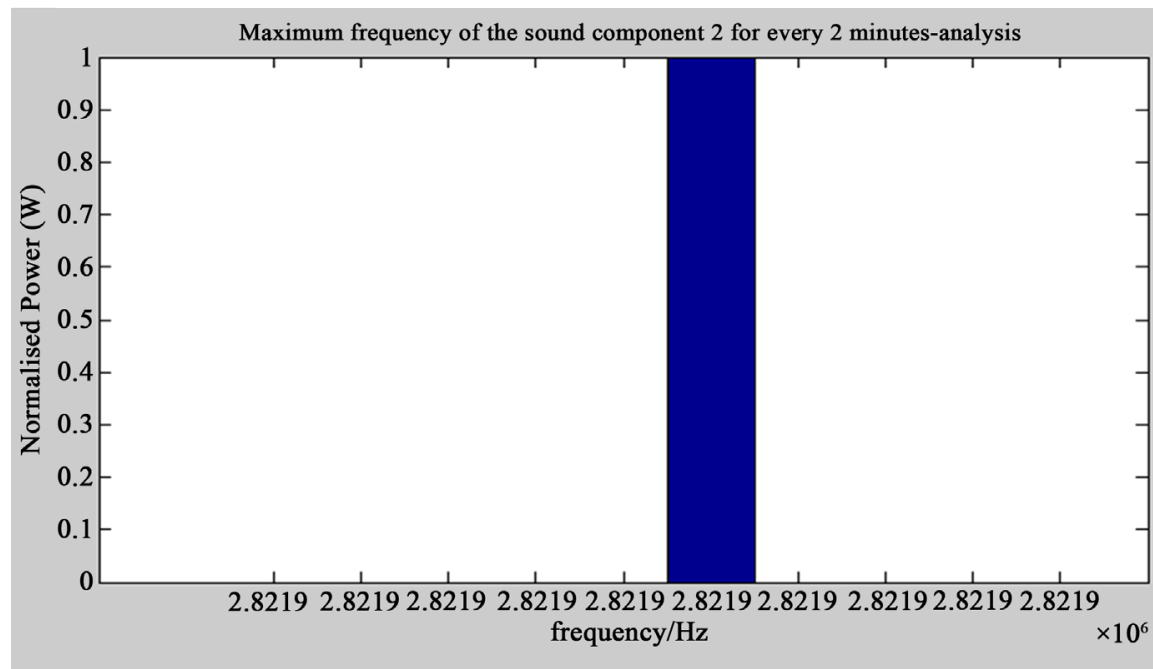

Figure 10. The frequency component emanated from the Fast Fourier transform (FFT) of the signal component 2 showed a maximum peak at $2.8219 \mathrm{MHz}$ for the sound component 2 and this is the average for every 2 minutes for the sound wav file.

1) Ultra-Sound component 1: $2.9949 \times 10^{6} \mathrm{~Hz}$

2) Ultra-Sound component 2: $2.8219 \times 10^{6} \mathrm{~Hz}$

\section{Discussions}

This research analysed the sound waves generated from a sound device by $\mathrm{R}$. David Case and the sound is called after him which is R. David Case sound wave file. The sound has the capability to cure or accelerate the healing process or reverse the process of tinnitus. It is posited that there may be some frequencies that are found in the generated sound waves which could do this.

Subjectively, based on various feedbacks from people who were administered this sound (at their own consents) described the sounds as follows. One of the 
participant who listened to the long sound wave at low volume intermittently state that he does not need Valium to circumvent the ringing in the ear and he states that the amplitude of the tinnitus seems to fluctuate high and low with improved cognitive concentration. Another participant who listened to the sound continuously for couple of days explained that he is able to do his day to day activities in peace and he states that he is cured from the tinnitus. Another participant posits that the sound waves have the capability to eliminate vibrations that were occurring on chest area, and then torso and also he is looking forward to remove occurrences from his lower body. Another subject claimed that the sound therapy he received from this peculiar sound really stopped his hyperacusis [7] in minimum duration (couple of weeks) and this sound has reduced his tinnitus greatly. Currently, he strongly wants to get rid of the tinnitus by listening to the sounds more consistently than he did before. The next participant realises that after 7 consecutive days of listening 6 hours of the sounds daily, he has observed that his right ear which normally can hear up to $10,400 \mathrm{~Hz}$ improve to $14,600 \mathrm{~Hz}$. His specialist advised him to continue using such sound device as his tinnitus has greatly improved as well as his hearing capabilities. Last but not the least, a participant found that he can sleep better at night after listening to the sounds and at low volume.

Technically speaking, the production of the ultrasound waves can happen owing to two types of effects that are: the first way is the magnetostriction oscillator and the second way is the piezoelectric oscillator. In our case, we will study the magnetostriction oscillator as it represents closely the materials that are found in the headphone (coil + magnet). This is elaborated in further research conducted by the authors. Based on the digital signal analysis of the sound wav and the headphone frequency characteristics, it was found that there is a much higher ultrasound frequency which is being generated. This, in turn is causing a positive effect on those people suffering from tinnitus [8] [9].

\section{Conclusion}

In this case study research, it is demonstrated how the unique sound wave comprising of both stereo and mono-waves generated by R. David Case coupled with the headphone characteristics (based on its Power-frequency response) can in fact alleviate the tinnitus problem. The ultrasound frequencies, as obtained in this research, can reverse the process of creating tinnitus and therefore it heals the human beings suffering from it. Based on the different interviews and feedbacks, it is clear that the sound wav and the headphone can be treated as a medical sound treatment or medical ultrasound treatment. Further research will look at analysing a larger sample of subjects/participants with their consent and observe if there are no long-term side effects. Couple with that, we need to devise an anti-tinnitus administration protocol and state clearly in that protocol the duration of listening this sound, and at what ideal amplitude, should the sound be played. The sound can concentrate in specific range of frequencies. 


\section{Conflicts of Interest}

The authors declare no conflicts of interest regarding the publication of this paper.

\section{References}

[1] Hynynen, K. and Clement, G. (2007) Clinical Applications of Focused UltrasoundThe Brain. International Journal of Hyperthermia, 23, 193-202. https://doi.org/10.1080/02656730701200094

[2] Martin, E. and Werner, B. (2013) Focused Ultrasound Surgery of the Brain. Current Radiolology Reports, 1, 126-135. https://doi.org/10.1007/s40134-013-0013-0

[3] Didar Urynbassarova, Li, B.Z. and Zhang, Z.C. (2017) A Convolution Theorem for the Polynomial Fourier Transform. IAENG International Journal of Applied Mathematics, 47, 381-387.

[4] McGillem, C.D. and Cooper, G.R. (1984) Continuous and Discrete Signal and System Analysis. 2nd Edition, Holt, Rinehart and Winston, New York, 118 (3-102). ISBN 0-03-061703-0.

[5] Proakis, J.G. and Manolakis, D.G. (1996) Digital Signal Processing: Principles, Algorithms and Applications. 3rd Edition, Prentice-Hall International, New Jersey, 297.

[6] Pienkowski, M., Tyler, R.S., Roncancio, E.R., Jun, H.J., Brozoski, T., Dauman, N., Coelho, C.B., Andersson, G., Keiner, A.J., Cacace, A.T., Martin, N. and Moore, B.C. (2014) A Review of Hyperacusis and Future Directions: Part II. Measurement, Mechanisms, and Treatment. American Journal of Audiology, 23, 420-436. https://doi.org/10.1044/2014 AJA-13-0037

[7] McCombe, A., Baguley, D., Coles, R., McKenna, L., McKinney, C. and WindleTaylor, P. (2001) Guidelines for the Grading of Tinnitus Severity: The Results of a Working Group Commissioned by the British Association of Otolaryngologists, Head and Neck Surgeons, 1999. Clinical Otolaryngology and Allied Sciences, 26, 388-393. https://doi.org/10.1046/j.1365-2273.2001.00490.x

[8] Langguth, B., Goodey, R., Azevedo, A., et al. (2007) Consensus for Tinnitus Patient Assessment and Treatment Outcome Measurement: Tinnitus Research Initiative meeting, Regensburg, July 2006. Progress in Brain Research, 166, 525-536. https://doi.org/10.1016/S0079-6123(07)66050-6

[9] Meikle, M.B., Stewart, B.J., Griest, S.E., et al. (2007) Assessment of Tinnitus: Measurement of Treatment Outcomes. Progress in Brain Research, 166, 511-521. https://doi.org/10.1016/S0079-6123(07)66049-X 